\title{
Copper-Catalyzed Domino Halide Exchange-Cyanation of Aryl Bromides
}

\author{
Jacopo Zanon, Artis Klapars and Stephen L. Buchwald* \\ Contribution from the Department of Chemistry, Massachusetts Institute of Technology, \\ Cambridge, Massachusetts 02139
}

\section{Supporting Information}

\section{General Considerations}

Reagents: Sodium cyanide (CAUTION: HIGHLY TOXIC) ${ }^{1}$ was purchased from Aldrich (97\% pure). Copper(I) iodide (fine powder) was purchased from Strem (98\% pure). If granulated $\mathrm{CuI}$, available from other sources, is used instead, it may be necessary to grind it. CuI is air-stable and does not require any special precautions other than storage in an amber vial. $N, N$ '-Dimethylethylenediamine was purchased from Aldrich. It forms a carbonate salt if exposed to air although we did not encounter any reproducibility problems even when using old samples of the diamine that had turned light brown and contained some precipitate. Potassium iodide (99.9\% pure, powder) was purchased from Alfa Aesar and stored in a dessicator. Although KI and $\mathrm{NaCN}$ were weighed out in the air, care was taken to minimize exposure to air due to the hygroscopicity of the salts, particularly during very humid periods of the year. Toluene was purchased from J. T. Baker in CYCLE-TAINER ${ }^{2}$ solvent delivery kegs, which were purged with argon for $2 \mathrm{~h}$ and purified by passing the toluene through two packed columns of neutral alumina and copper (II) oxide under argon pressure. All other reagents were commercially available and used without further purification. Flash column chromatography was performed with J. T. Baker silica gel 60 (230-400 mesh).

\footnotetext{
${ }^{1}$ Cyanide containing waste can be decontaminated using a solution of $\mathrm{NaOCl}$ in water (CLOROX bleach). See: Lunn, G.; Sansone, E. B. Destruction of Hazardous Chemicals in the Laboratory, 2nd ed., Wiley \& Sons: New York, 1994; pp 133-138.

2 (a) Pangborn, A. B.; Giardello, M. A.; Grubbs, R. H.; Rosen, R. K.; Timmers, F. J. Organometallics 1996, 15, 1518-1520. (b) Alaimo, P. J.; Peters, D. W.; Arnold, J.; Bergman, R. G. J. Chem. Ed. 2001, 78, 64.
} 
Techniques: The copper-catalyzed cyanation reactions are sensitive to oxygen and moisture. Nevertheless, neither glovebox techniques nor purification of the commercially available reagents are required. The following procedure was used for the reactions that were performed in Schlenk tubes. After a $15 \mathrm{~mL}$ Schlenk tube with a screw thread (Kontes) was dried in an oven at $120{ }^{\circ} \mathrm{C}$ overnight, it was equipped with a $10 \times 3 \mathrm{~mm}$ Teflon-coated stirring bar and a Teflon valve, evacuated, then backfilled with argon. The solid reagents were weighed out in the air by adding them directly to the Schlenk tube with the Teflon valve removed. The Schlenk tube was again fitted with the Teflon valve, evacuated and backfilled with argon. Under a positive pressure of argon, the Teflon valve was replaced with a rubber septum, and the liquid reagents were added to the Schlenk tube using Hamilton mycrosyringes (if $<500 \mu \mathrm{L}$ ) or all polypropylene/polyethylene disposable syringes (if $>500 \mu \mathrm{L}$ ). The rubber septum was replaced with a Teflon valve under positive pressure of argon. The Schlenk tube was sealed and heated in an oil bath for the specified time while stirring at the appropriate stirring rate. The stirring rate must be set carefully to avoid deposition of the solid on the walls of the Schlenk tube and to ensure at the same time an effective mixing.

Analytical methods: IR spectra were recorded on a Perkin-Elmer FT-IR 2000. Elemental analyses were performed by Atlantic Microlabs, Inc., Norcross, GA. ${ }^{1} \mathrm{H}$ NMR and ${ }^{13} \mathrm{C}$ NMR spectra were recorded on a Bruker $400 \mathrm{MHz}$ instrument with chemical shifts reported relative to residual deuterated solvent peaks or tetramethylsilane internal standard. Gas chromatographic analysis was performed on an Agilent 6890 instrument with an FID detector and an Agilent $10 \mathrm{~m}$ $\times 0.10 \mu \mathrm{m}$ i.d. HP-1 capillary column. Mass spectra (GC/MS) were recorded on a Hewlett Packard model GCD. All yields reported in Table 1 and Tale 2 refer to isolated yields (average of two runs) of compounds estimated to be $>95 \%$ pure as determined by ${ }^{1} \mathrm{H}$ NMR and GC. The procedures described in this section are representative, and thus the yields for the individual reactions may differ slightly from the average yields reported in Table 1 and Table 2. 


\section{Copper-Catalyzed Conversion of Aryl Bromides into the Corresponding Cyanides}

General Procedure. A Schlenk tube was charged with NaCN (102 mg, $2.08 \mathrm{mmol}), \mathrm{CuI}$ (33 mg, $0.17 \mathrm{mmol}, 10 \mathrm{~mol} \%$ ), aryl bromide (if it is a solid at room temperature; $1.74 \mathrm{mmol}$ ), and $\mathrm{KI}$ (57 $\mathrm{mg}, 0.34 \mathrm{mmol}, 20 \mathrm{~mol} \%$ ). The tube was then briefly evacuated and backfilled with argon three times. Anhydrous toluene $(1.2 \mathrm{~mL}), N, N$-dimethylethylenediamine $(185 \mu \mathrm{L}, 1.74 \mathrm{mmol})$, and aryl bromide (if it is a liquid at room temperature; $1.74 \mathrm{mmol}$ ) were added under argon. The Schlenk tube was sealed with a Teflon valve and the reaction mixture was stirred at $110{ }^{\circ} \mathrm{C}$ for 22-24 h. The resulting suspension was allowed to reach room temperature, diluted with $30 \%$ aq ammonia $(3 \mathrm{~mL})$, and extracted with ethyl acetate $(4 \times 2 \mathrm{~mL})$. The combined organic phases were dried $\left(\mathrm{MgSO}_{4}\right.$ or $\left.\mathrm{Na}_{2} \mathrm{SO}_{4}\right)$, concentrated, and the residue was purified by flash chromatography on silica gel to provide the desired product.

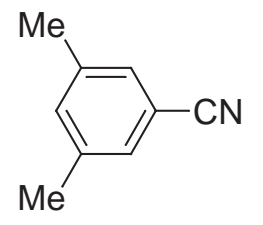

\section{3,5-Dimethylbenzonitrile (Table 1, Entry 1)}

An oven dried $100 \mathrm{~mL}$ three necked round bottom flask was charged, under positive pressure of argon, with $\mathrm{NaCN}$ (2.04 g, $41.6 \mathrm{mmol}) \mathrm{CuI}$ (660 mg, $3.47 \mathrm{mmol}, 10 \mathrm{~mol} \%$ ), KI (1.14 g, 6.87 mmol, $20 \mathrm{~mol} \%)$, and anhydrous toluene $(25 \mathrm{~mL}) . N, N^{\prime}$-Dimethylethylenediamine $(3.7 \mathrm{~mL}, 35$ $\mathrm{mmol})$ and 5-bromo- $m$-xylene $(4.7 \mathrm{~mL}, 35 \mathrm{mmol})$ were added dropwise under mechanical stirring. The reaction mixture was stirred at $110^{\circ} \mathrm{C}$ for $24 \mathrm{~h}$. The resulting suspension was allowed to reach room temperature, diluted with $30 \%$ aq ammonia $(20 \mathrm{~mL})$ and extracted with toluene $(2 \times 10$ $\mathrm{mL}$ ). The combined organic phases were dried over $\mathrm{MgSO}_{4}$, concentrated, and the residue was purified by distillation under reduced pressure (bp $100{ }^{\circ} \mathrm{C} @ 10$ Torr) to provide the desired product as a white crystalline solid (4.08 g, 90\% yield). Mp $42-43{ }^{\circ} \mathrm{C}$ (lit., ${ }^{3} 42.9{ }^{\circ} \mathrm{C}$ ). ${ }^{1} \mathrm{H}$ NMR (lit.; $400 \mathrm{MHz}, \mathrm{CDCl}_{3}$ ): $\delta 7.27$ (s, 2H), $7.23(\mathrm{~s}, 1 \mathrm{H}), 2.36(\mathrm{~s}, 6 \mathrm{H}) ;{ }^{13} \mathrm{C} \mathrm{NMR}\left(100 \mathrm{MHz}, \mathrm{CDCl}_{3}\right.$ ): 139.4, 135.0, 130.1, 119.7, 112.4, 21.5; IR (neat, $\mathrm{cm}^{-1}$ ): 2230, 1605, 1378, 907, 854, 682. Anal. Calcd. for $\mathrm{C}_{9} \mathrm{H}_{9} \mathrm{~N}$ : C, 82.41; H, 6.92; N, 10.68. Found: C, 82.11; H, 6.88, N, 10.52.

\footnotetext{
${ }^{3}$ Birch, S. F.; Dean, R. A.; Fidler, F. A.; Lowry, R. A. J. Am. Chem. Soc. 1949, 71, 1362.

${ }^{4}$ Nomura, Y.; Takeuchi, Y. Tetrahedron 1969, 25, 3825.
} 


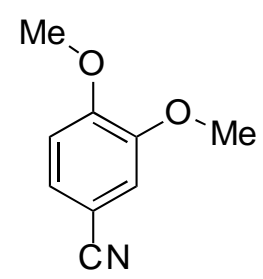

\section{3,4-Dimethoxy-benzonitrile (Table 1, Entry 2)}

A Schlenk tube was charged with $\mathrm{NaCN}$ (204 mg, $4.16 \mathrm{mmol}), \mathrm{CuI}$ (66 mg, $0.35 \mathrm{mmol}, 10$ $\mathrm{mol} \%$ ), and KI (114 mg, $0.687 \mathrm{mmol}, 20 \mathrm{~mol} \%$ ), briefly evacuated and backfilled with argon three times. Anhydrous toluene $(2.4 \mathrm{~mL}), N, N$ '-dimethylethylenediamine $(370 \mu \mathrm{L}, 3.48 \mathrm{mmol})$, and 4bromoveratrole $(500 \mu \mathrm{L}, 3.46 \mathrm{mmol})$ were added under argon. The Schlenk tube was sealed with a Teflon valve and the reaction mixture was stirred at $110{ }^{\circ} \mathrm{C}$ for $24 \mathrm{~h}$. The resulting suspension was allowed to reach room temperature, diluted with $30 \%$ aq ammonia $(2 \mathrm{~mL})$, and extracted with ethyl acetate $(4 \times 4 \mathrm{~mL})$. The combined organic phases were dried over $\mathrm{MgSO}_{4}$, concentrated, and the residue was purified by distillation at reduced pressure (bp $160{ }^{\circ} \mathrm{C} @ 1$ Torr) to provide the desired product as pale yellow solid (500 mg, 91\% yield). Mp 60-62 ${ }^{\circ} \mathrm{C}$ (lit. ${ }^{5} 63.0-63.5{ }^{\circ} \mathrm{C}$ ). ${ }^{1} \mathrm{H}$ NMR (lit.; $400 \mathrm{MHz}, \mathrm{CDCl}_{3}, J$ values are reported in $\mathrm{Hz}$ ): $\delta 7.29$ (dd, $J=8.3, J=2.0,1 \mathrm{H}$ ), 7.08 $(\mathrm{d}, J=2.0,1 \mathrm{H}), 6.91(\mathrm{~d}, J=8.3,1 \mathrm{H}), 3.94(\mathrm{~s}, 3 \mathrm{H}), 3.91(\mathrm{~s}, 3 \mathrm{H}) ;{ }^{13} \mathrm{C} \mathrm{NMR}\left(100 \mathrm{MHz}, \mathrm{CDCl}_{3}\right): \delta$ 153.2, 149.6, 126.9, 119.7, 114.3, 111.6, 104.3, 56.54, 56.51; IR (neat, $\mathrm{cm}^{-1}$ ): 2225, 1598, 1583, 1519, 1245, 1158, 1139, 1018, 876, 811, 617. Anal. Calcd. for $\mathrm{C}_{9} \mathrm{H}_{9} \mathrm{NO}_{2}$ : C, 66.25; H, 5.56; N, 8.58. Found: C, 66.02; H, 5.72; N, 8.69.

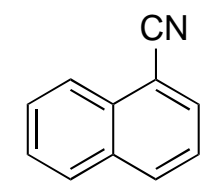

\section{Naphthalene-1-carbonitrile (Table 1, Entry 3)}

A Schlenk tube was charged with $\mathrm{NaCN}$ (95 mg, $1.94 \mathrm{mmol}), \mathrm{CuI}$ (31 mg, $0.16 \mathrm{mmol}, 10 \mathrm{~mol} \%$ ), and KI (54 mg, $0.33 \mathrm{mmol}, 20 \mathrm{~mol} \%$ ), briefly evacuated and backfilled with argon three times. Anhydrous toluene $(1.2 \mathrm{~mL}), N, N$ '-dimethylethylenediamine $(175 \mu \mathrm{L}, 1.64 \mathrm{mmol})$, and 1bromonaphthalene $(225 \mu \mathrm{L}, 1.62 \mathrm{mmol})$ were added under argon. The Schlenk tube was sealed with a Teflon valve and the reaction mixture was stirred at $130{ }^{\circ} \mathrm{C}$ for $24 \mathrm{~h}$. The resulting suspension was allowed to reach room temperature, diluted with $30 \%$ aqueous ammonia ( $2 \mathrm{~mL}$ ) and extracted with ethyl acetate $(4 \times 2 \mathrm{~mL})$. The combined organic phases were dried over

\footnotetext{
${ }^{5}$ Murahashi, S.-I.; Naota, T.; Nakajima, N. J. Org. Chem. 1986, 51, 898.
} 
$\mathrm{MgSO}_{4}$, concentrated, and the residue was purified by flash chromatography on silica gel (hexane/ethyl acetate 20:1) to provide the desired product as a pale yellow solid (234 $\mathrm{mg}, 94 \%$ yield). Mp 35-36 ${ }^{\circ} \mathrm{C}$ (lit., ${ }^{6} 37^{\circ} \mathrm{C}$ ). ${ }^{1} \mathrm{H} \mathrm{NMR}$ (lit.; ${ }^{7} 400 \mathrm{MHz}, \mathrm{CDCl}_{3}, J$ values are reported in $\mathrm{Hz}): \delta 8.27(\mathrm{~d}, J=8.4,1 \mathrm{H}), 8.11(\mathrm{~d}, J=8.3,1 \mathrm{H}), 7.96(\mathrm{~d}, J=8.1,1 \mathrm{H}), 7.94(\mathrm{dd}, J=7.2, J=1.1$, $1 \mathrm{H}), 7.73(\mathrm{ddd}, J=8.3, J=6.9, J=1.34,1 \mathrm{H}), 7.65(\mathrm{ddd}, J=8.3, J=7.1, J=1.2,1 \mathrm{H}), 7.55(\mathrm{dd}, J=$ 8.3, $J=7.1,1 \mathrm{H}) ;{ }^{13} \mathrm{C} \mathrm{NMR}\left(100 \mathrm{MHz}, \mathrm{CDCl}_{3}\right)$ : 133.7, 133.2, 133.1, 132.8, 129.1, 129.0, 128.0, 125.6, 125.4, 118.3, 110.6; IR (neat, $\mathrm{cm}^{-1}$ ): 2222, 1604, 1513, 1376, 855, 802, 772, 684, 451. Anal. Calcd. for $\mathrm{C}_{11} \mathrm{H}_{7} \mathrm{~N}$ : C, 86.25; H, 4.61; N, 9.14. Found: C, 86.04; H 4.61; N 9.05.

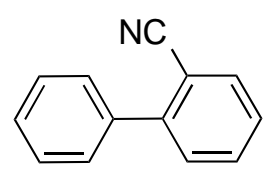

\section{Biphenyl-2-carbonitrile (Table 1, Entry 4)}

A Schlenk tube was charged with $\mathrm{NaCN}$ (98 mg, $2.0 \mathrm{mmol}), \mathrm{CuI}(31 \mathrm{mg}, 0.16 \mathrm{mmol}, 10 \mathrm{~mol} \%$ ), and KI (55 mg, $0.33 \mathrm{mmol}, 20 \mathrm{~mol} \%$ ), briefly evacuated and backfilled with argon three times. Anhydrous toluene $(1.2 \mathrm{~mL}), N, N^{\prime}$-dimethylethylenediamine $(175 \mu \mathrm{L}, 1.64 \mathrm{mmol})$, and 2bromobiphenyl $(285 \mu \mathrm{L}, 1.65 \mathrm{mmol})$ were added under argon. The Schlenk tube was sealed with a Teflon valve and the reaction mixture was stirred at $130{ }^{\circ} \mathrm{C}$ for $24 \mathrm{~h}$. The resulting suspension was allowed to reach room temperature, diluted with $30 \%$ aq ammonia $(2 \mathrm{~mL})$, and extracted with ethyl acetate $(4 \times 2 \mathrm{~mL})$. The combined organic phases were dried over $\mathrm{MgSO}_{4}$, concentrated, and the residue was purified by flash chromatography on silica gel (hexane-ethyl acetate 20:1) to provide the desired product as a light yellow oil which crystallized upon storing in a refrigerator (289 mg, 98\% yield). Mp 34-37 ${ }^{\circ} \mathrm{C}$ (lit., ${ }^{8} 35-37{ }^{\circ} \mathrm{C}$ ). ${ }^{1} \mathrm{H}$ NMR (lit.; ${ }^{9} 400 \mathrm{MHz}, \mathrm{CDCl}_{3}, J$ values are reported in Hz): $\delta 7.80(\mathrm{ddd}, J=7.8, J=1.5, J=0.5,1 \mathrm{H}), 7.68(\mathrm{td}, J=7.8, J=1.3,1 \mathrm{H}), 7.61$ 7.44 (m, 7H); ${ }^{13} \mathrm{C} \mathrm{NMR} \mathrm{(100} \mathrm{MHz,} \mathrm{CDCl}_{3}$ ): 145.9, 138.6, 138.5, 134.2, 133.3, 130.5, 129.2, 129.1, 128.0, 119.2, 111.7; IR (neat, $\mathrm{cm}^{-1}$ ): 2224, 1597, 1500, 1477, 1451, 1433, 759, 735, 700. Anal. Calcd. for $\mathrm{C}_{13} \mathrm{H}_{9} \mathrm{~N}$ : C, 87.12; H, 5.06; N, 7.82. Found: C 86.84; H 5.22; N 7.94.

\footnotetext{
${ }^{6}$ Blum, J.; Oppenheimer, E.; Bergmann, E. J. Am. Chem. Soc. 1967, 89, 2338.

${ }^{7}$ Abraham, R. J.; Reid, M. Magn. Reson. Chem. 2000, 38, 570.

${ }^{8}$ Sain, B.; Sandhu, J. S. J. Org. Chem. 1990, 55, 2545.

${ }^{9}$ Hassan, J.; Hathroubi, C.; Gozzi, C.; Lemaire, M. Tetrahedron 2001, 57, 7845.
} 


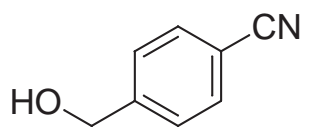

\section{4-Hydroxymethylbenzonitrile (Table 1, Entry 5)}

Following the general procedure, 4-bromophenylmethanol $(325 \mathrm{mg}, 1.74 \mathrm{mmol})$ was converted into 4-hydroxymethylbenzonitrile in $20 \mathrm{~h}$ at $110{ }^{\circ} \mathrm{C}$. Purification of the crude product by column chromatography on silica gel (hexane/ethyl acetate 2:1) provided the desired product as a white crystalline solid (190 mg, 83\% yield). Mp 39-40 ${ }^{\circ} \mathrm{C}$ (lit., ${ }^{10} 39-41{ }^{\circ} \mathrm{C}$ ). ${ }^{1} \mathrm{H}$ NMR (lit.; ${ }^{10} 400$ $\mathrm{MHz}, \mathrm{CDCl}_{3}, J$ values are reported in $\mathrm{Hz}$ ): $\delta 7.66$ (dt, $\left.J=8.1, J=1.8,2 \mathrm{H}\right), 7.49(\mathrm{~d}, J=8.1,2 \mathrm{H}$ ), 4.80 (s, 2H), 2.15 (bs, $1 \mathrm{H}) ;{ }^{13} \mathrm{C}$ NMR (100 MHz, $\mathrm{CDCl}_{3}$ ): 146.6, 132.7, 127.4, 119.3, 111.5, 64.6; IR (neat, $\mathrm{cm}^{-1}$ ): 3484, 2233, 1609, 1428, 1208, 1030, 847, 820, 566. Anal. Calcd. for $\mathrm{C}_{8} \mathrm{H}_{7} \mathrm{NO}: \mathrm{C}$, 72.16; H, 5.30; N, 10.52. Found: C 72.15; H 5.33; N 10.44<smiles>CCOC(=O)Cc1ccc(C#N)cc1</smiles>

\section{Ethyl 4-Cyanophenylacetate (Table 1, Entry 6)}

Following the general procedure, ethyl 4-bromophenylacetate (417 $\mathrm{mg}, 1.72 \mathrm{mmol}$ ) was converted into ethyl 4-cyanophenylacetate in $24 \mathrm{~h}$ at $130{ }^{\circ} \mathrm{C}$. Purification of the crude product by column chromatography on silica gel (hexane/ethyl acetate 10:1) provided the desired product as a fine white powder $\left(230 \mathrm{mg}, 70 \%\right.$ yield). Mp 86-87 ${ }^{\circ} \mathrm{C}$ (lit., $\left.{ }^{11} 87-88{ }^{\circ} \mathrm{C}\right) .{ }^{1} \mathrm{H}$ NMR (lit.; ${ }^{12}$ $400 \mathrm{MHz}, \mathrm{CDCl}_{3}, J$ values are reported in $\left.\mathrm{Hz}\right): \delta 7.63(\mathrm{dt}, J=8.2, J=1.8,2 \mathrm{H}), 7.42(\mathrm{~d}, J=8.2$, $2 \mathrm{H}), 4.18$ (q, $J=7.0,2 \mathrm{H}), 3.69$ (s, 2H), 1.27 (t, $J=7.0,3 \mathrm{H}) ;{ }^{13} \mathrm{C}$ NMR $\left(100 \mathrm{MHz}, \mathrm{CDCl}_{3}\right): \delta$ 170.8, 139.9, 132.7, 130.6, 119.2, 111.5, 61.7, 41.7, 14.6; IR (neat, $\mathrm{cm}^{-1}$ ): 2231, 1734, 1421, 1222, 1176, 1028. Anal. Calcd. for $\mathrm{C}_{11} \mathrm{H}_{11} \mathrm{NO}_{2}$ : C, 69.83; H, 5.86; N, 7.40. Found: C 69.69; H 5.93; N 7.24.

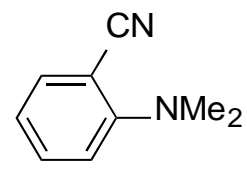

\section{2-Dimethylaminobenzonitrile (Table 1, Entry 7)}

Following the general procedure, $N, N$-dimethyl-2-bromoaniline $(250 \mu \mathrm{L}, 1.75 \mathrm{mmol})$ was converted into 2-dimethylaminobenzonitrile in $24 \mathrm{~h}$ at $130{ }^{\circ} \mathrm{C}$. Purification of the crude product

\footnotetext{
${ }^{10}$ Yoon, N. M.; Pak, C. S.; Brown, H. C.; Krishnamurthy, S.; Stocky, T. P. J. Org. Chem. 1973, $38,2786$.

${ }^{11}$ Norman, R. O. C.; Ralph, P. D. J. Chem. Soc. 1963, 5431.

${ }^{12}$ Beugelmans, R.; Bois-Choussy, M.; Boudet, B. Tetrahedron, 1982, 38, 3479.
} 
by column chromatography on silica gel (hexane/ethyl acetate 10:1) provided the desired product as a pale yellow oil (225 mg, 88\% yield). ${ }^{1} \mathrm{H}$ NMR (lit. ${ }^{13} 400 \mathrm{MHz}, \mathrm{CDCl}_{3}, J$ values are reported in Hz): $\delta 7.54$ (ddd, $J=7.7, J=1.7, J=1.0,1 \mathrm{H}$ ), 7.44 (ddd, $J=9.00, J=7.3, J=1.7,1 \mathrm{H}$ ), 6.93 (bd, $J=8.5,1 \mathrm{H}), 6.87(\mathrm{td}, J=7.3, J=1.0,1 \mathrm{H}) ;{ }^{13} \mathrm{C} \mathrm{NMR}\left(100 \mathrm{MHz}, \mathrm{CDCl}_{3}\right): \delta 155.7,135.4$, 133.9, 120.1, 119.6, 117.2, 101.7, 43.5; IR (neat, $\mathrm{cm}^{-1}$ ): 2215, 1599, 1499, 1433, 948, 756.

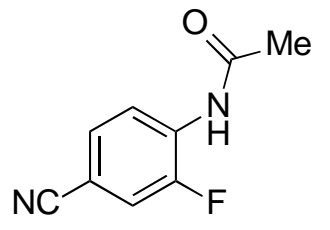

\section{$\mathrm{N}$-(4-Cyano-2-fluorophenyl)acetamide (Table 1, Entry 8)}

A Schlenk tube was charged with $\mathrm{NaCN}$ (137 mg, $2.80 \mathrm{mmol}$ ), CuI (44 mg, $0.23 \mathrm{mmol}, 10$ mol\%), KI (77 mg, $0.46 \mathrm{mmol}, 20 \mathrm{~mol} \%$ ), and $N$-(4-bromo-2-fluorophenyl)acetamide (540 $\mathrm{mg}$, $2.33 \mathrm{mmol})$, briefly evacuated and backfilled with argon three times. Anhydrous toluene (1.2 $\mathrm{mL})$ and $N, N$ '-dimethylethylenediamine $(250 \mu \mathrm{L}, 2.35 \mathrm{mmol})$ were added under argon. The Schlenk tube was sealed with a Teflon valve and the reaction mixture was stirred at $110{ }^{\circ} \mathrm{C}$ for 24 h. The resulting suspension was allowed to reach room temperature, diluted with $30 \%$ aq ammonia $(2 \mathrm{~mL})$, and extracted with ethyl acetate $(4 \times 2 \mathrm{~mL})$. The combined organic phases were dried over $\mathrm{MgSO}_{4}$, concentrated, and the residue was purified by flash chromatography on silica gel (hexane/ethyl acetate 1:1) to provide the desired product as a fine white powder (337 $\mathrm{mg}$, $87 \%$ yield). Mp 169.5-171.5 ${ }^{\circ} \mathrm{C} ;{ }^{1} \mathrm{H} \mathrm{NMR}\left(400 \mathrm{MHz}, \mathrm{CDCl}_{3}, J\right.$ values are reported in $\mathrm{Hz}$ ): $\delta 8.59$ (t, $J=8.4,1 \mathrm{H}), 7.63(\mathrm{bs}, 1 \mathrm{H}), 7.48(\mathrm{~d}, J=8.4,1 \mathrm{H}), 7.41(\mathrm{dd}, J=10.6, J=1.8,1 \mathrm{H}), 2.30(\mathrm{~s}, 3 \mathrm{H})$; ${ }^{13} \mathrm{C}$ NMR (100 MHz, $\left.\mathrm{CDCl}_{3}\right): 169.0,151.3(\mathrm{~d}, J=244 \mathrm{~Hz}), 131.6(\mathrm{~d}, J=9.6 \mathrm{~Hz}), 129.9$ (d, $J=3.5$ $\mathrm{Hz}), 121.9,118.7(\mathrm{~d}, J=22.8 \mathrm{~Hz}), 118.1(\mathrm{~d}, J=2.9 \mathrm{~Hz}), 107.1(\mathrm{~d}, J=9.3 \mathrm{~Hz}), 25.3$; IR (neat, $\mathrm{cm}^{-}$ $\left.{ }^{1}\right)$ : 3317, 2235, 1699, 1593, 1515, 834, 707. Anal. Calcd. for $\mathrm{C}_{9} \mathrm{H}_{7} \mathrm{FN}_{2} \mathrm{O}$ : C, 60.67; H, 3.96; N, 15.72. Found: C $60.42 ; \mathrm{H} 3.94 ; \mathrm{N} 15.63$.<smiles>CC(=O)Nc1ccc(C)cc1C#N</smiles>

\section{N-(2-Cyano-4-methylphenyl)acetamide (Table 1, Entry 9)}

Following the general procedure, 2-bromo-4-methylacetanilide (395 $\mathrm{mg}, 1.74 \mathrm{mmol}$ ) was converted into $N$-(2-cyano-4-methylphenyl)acetamide in $24 \mathrm{~h}$ at $130^{\circ} \mathrm{C}$. Purification of the crude

\footnotetext{
${ }^{13}$ Gupton, J. T.; Idoux, J. P.; Baker, G.; Colon, C.; Crews, A.D.; Jurss, C.D.; Rampi, R. C. J. Org. Chem.
} 
product by column chromatography on silica gel (hexane/ethyl acetate 1:1) provided the desired product as a pale yellow crystalline powder (200 mg, 70\% yield). Mp 133-135 ${ }^{\circ} \mathrm{C}$. ${ }^{1} \mathrm{H}$ NMR $\left(400 \mathrm{MHz}, \mathrm{CDCl}_{3}, J\right.$ values are reported in $\left.\mathrm{Hz}\right): \delta 8.24(\mathrm{~d}, J=8.3,1 \mathrm{H}), 7.65(\mathrm{bs}, 1 \mathrm{H}), 7.40(\mathrm{~d}, J=$ 8.3, 1H), 7.39 (s, 1H), $2.34(\mathrm{~s}, 3 \mathrm{H}), 2.26(\mathrm{~s}, 3 \mathrm{H}) ;{ }^{13} \mathrm{C} \mathrm{NMR}\left(100 \mathrm{MHz}, \mathrm{CDCl}_{3}\right)$ : 169.0, 138.5, 135.4, 134.7, 132.6, 122.0, 117.0, 102.4, 25.1, 20.9; IR (neat, $\mathrm{cm}^{-1}$ ): 3253, 2225, 1665, 1589, 1535, 1304, 1275, 1157, 828, 678, 497. Anal. Calcd. for $\mathrm{C}_{10} \mathrm{H}_{10} \mathrm{~N}_{2} \mathrm{O}$ : C, 68.95; H, 5.79; N, 16.08 . Found: C, 69.10; H, 5.90; N, 15.97.

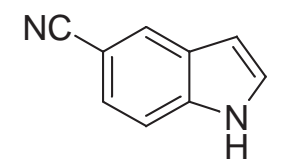

\section{$1 H$-Indole-5-carbonitrile (Table 2, Entry 1)}

Following the general procedure, 5-bromoindole $(340 \mathrm{mg}, 1.74 \mathrm{mmol})$ was converted into $1 H$ indole-5-carbonitrile in $24 \mathrm{~h}$ at $110{ }^{\circ} \mathrm{C}$. Purification of the crude product by column chromatography on silica gel (hexane/ethyl acetate 6:1) provided the desired product as a light brown solid (190 mg, 80\% yield). Mp 104-105 ${ }^{\circ} \mathrm{C}$ (lit., ${ }^{14} 104-106{ }^{\circ} \mathrm{C}$ ). ${ }^{1} \mathrm{H}$ NMR (lit. $;^{15} 400$ $\mathrm{MHz}, \mathrm{CDCl}_{3}, J$ values are reported in $\mathrm{Hz}$ ): $\delta 8.72(\mathrm{bs}, 1 \mathrm{H}), 8.03(\mathrm{~s}, 1 \mathrm{H}), 7.50(\mathrm{~d}, J=8.4,1 \mathrm{H}), 7.45$ $(\mathrm{dd}, J=8.4, J=1.5,1 \mathrm{H}), 7.38(\mathrm{t}, J=2.8,1 \mathrm{H}), 6.68-6.64(\mathrm{~m}, 1 \mathrm{H}) ;{ }^{13} \mathrm{C} \mathrm{NMR}\left(100 \mathrm{MHz}, \mathrm{CDCl}_{3}\right): \delta$ 137.9, 128.1, 126.9, 126.8, 125.3, 121.3, 112.4, 103.9, 103.2; IR (neat, $\mathrm{cm}^{-1}$ ): 3399, 2226, 1612, 1470, 1418, 1347, 1089, 894. Anal. Calcd. for $\mathrm{C}_{9} \mathrm{H}_{6} \mathrm{~N}_{2}$ : C, 76.04; H, 4.25; N, 19.71. Found: C, $76.21 ; \mathrm{H}, 4.28 ; \mathrm{N}, 19.52$.

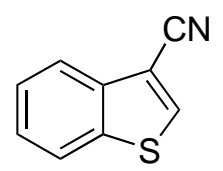

\section{Benzo[b]thiophene-3-carbonitrile (Table 2, Entry 2)}

Following the general procedure, 3-bromothianaphthene $(225 \mu \mathrm{L}, 1.72 \mathrm{mmol})$ was converted into benzo[b]thiophene-3-carbonitrile in $24 \mathrm{~h}$ at $110^{\circ} \mathrm{C}$. Purification of the crude product by column chromatography on silica gel (hexane/ethyl acetate 8:1) provided the desired product as a pale yellow solid (200 mg, 73\% yield). Mp 67-69 ${ }^{\circ} \mathrm{C}$ (lit., ${ }^{16} 70-71{ }^{\circ} \mathrm{C}$ ). ${ }^{1} \mathrm{H}$ NMR (lit.; ${ }^{16} 400 \mathrm{MHz}$, $\mathrm{CDCl}_{3}, J$ values are reported in $\left.\mathrm{Hz}\right): \delta 8.15(\mathrm{~s}, 1 \mathrm{H}), 8.03(\mathrm{~d}, J=7.8,1 \mathrm{H}), 7.94(\mathrm{~d}, J=7.8,1 \mathrm{H}), 7.58$ $(\mathrm{td}, J=8.1, J=1.0,1 \mathrm{H}), 7.52(\mathrm{dt}, J=7.1, J=1.0,1 \mathrm{H}) ;{ }^{13} \mathrm{C} \mathrm{NMR}\left(100 \mathrm{MHz}, \mathrm{CDCl}_{3}\right): \delta 138.9$,

1983, 48, 2933.

${ }^{14}$ Lindwall, H. G.; Mantell, G. J. J. Org. Chem. 1953, 18, 345.

${ }^{15}$ Morales-Rios, M. S.; del Rio, R. E.; Joseph-Nathan, P. Magn. Reson. Chem. 1989, 27, 1039.

${ }^{16}$ Yoshida, K.; Miyoshi, K. J. Chem. Soc., Perkin Trans. 1 1992, 333. 
138.0, 137.7, 126.6, 126.4, 123.3, 122.9, 114.8, 107.5; IR (neat, $\mathrm{cm}^{-1}$ ): 3108, 2224, 1462, 1426, 1256, 857, 814, 755, 729, 445. Anal. Calcd. for $\mathrm{C}_{9} \mathrm{H}_{5} \mathrm{NS}$ : C, 67.90; H, 3.17; N, 8.80. Found: C, 67.69; H, 3.11; N, 8.62.

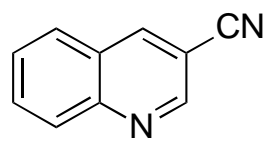

\section{Quinoline-3-carbonitrile (Table 2, Entry 3)}

Following the general procedure, 3-bromoquinoline $(235 \mu \mathrm{L}, 1.73 \mathrm{mmol})$ was converted into quinoline-3-carbonitrile in $24 \mathrm{~h}$ at $110{ }^{\circ} \mathrm{C}$. Purification of the crude product by column chromatography on silica gel (hexane/ethyl acetate 6:1) provided the desired product as a white crystalline powder (205 mg, 75\% yield). Mp 105-107 ${ }^{\circ} \mathrm{C}$ (lit., ${ }^{17} 105-107{ }^{\circ} \mathrm{C}$ ). ${ }^{1} \mathrm{H}$ NMR (lit.; ${ }^{17}$ $400 \mathrm{MHz}, \mathrm{CDCl}_{3}, J$ values are reported in $\left.\mathrm{Hz}\right): \delta 9.07(\mathrm{~d}, J=2.0,1 \mathrm{H}), 8.57(\mathrm{dd}, J=2.0, J=0.5$, $1 \mathrm{H}), 8.20(\mathrm{~d}, J=9.1,1 \mathrm{H}), 7.95-7.90(\mathrm{~m}, 2 \mathrm{H}), 7.73(\mathrm{t}, J=7.6,1 \mathrm{H}) ;{ }^{13} \mathrm{C} \mathrm{NMR}\left(100 \mathrm{MHz}, \mathrm{CDCl}_{3}\right): \delta$ 150.1, 149.2, 141.9, 133.2, 130.3, 128.9, 128.7, 126.6, 117.5, 107.0; IR (neat, $\mathrm{cm}^{-1}$ ): 2229, 1619, 1597, 1489, 1370, 1130, 982, 961, 923, 739, 747, 638, 474. Anal. Calcd. for $\mathrm{C}_{10} \mathrm{H}_{6} \mathrm{~N}_{2}$ : C, 77.91; H, 3.92; N, 18.17. Found: C, 77.96; H, 3.97; N, 18.32.

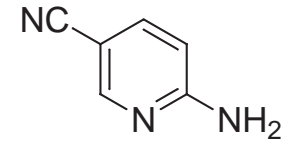

\section{6-Aminonicotinonitrile (Table 2, Entry 4)}

Following the general procedure, 2-amino-5-bromopyridine (294 mg, $1.70 \mathrm{mmol}$ ) was converted into 6-aminonicotinonitrile in $20 \mathrm{~h}$ at $110{ }^{\circ} \mathrm{C}$. Purification of the crude product by column chromatography on silica gel (hexane/ethyl acetate 2:5) provided the desired product as a white crystalline powder (180 mg, 90\% yield). Mp 160-162 ${ }^{\circ} \mathrm{C}$ (lit., ${ }^{18} 161-162{ }^{\circ} \mathrm{C}$ ). ${ }^{1} \mathrm{H}$ NMR (lit., ${ }^{19}$ $400 \mathrm{MHz}, \mathrm{CDCl}_{3}, J$ values are reported in $\left.\mathrm{Hz}\right): \delta 8.41-8.36(\mathrm{~m}, 1 \mathrm{H}), 7.64(\mathrm{dd}, J=8.6, J=2.2,1 \mathrm{H})$, $6.53(\mathrm{dd}, J=8.6, J=0.9,1 \mathrm{H}), 5.08(\mathrm{bs}, 2 \mathrm{H}) ;{ }^{13} \mathrm{C} \mathrm{NMR}\left(100 \mathrm{MHz}, \mathrm{CDCl}_{3}\right): 160.5,153.6,140.7$, 118.6, 108.4, 98.8; IR (neat, $\mathrm{cm}^{-1}$ ): 3414, 3136, 2211, 1654, 1601, 1509, 1410, 832, 546. Anal. Calcd. for $\mathrm{C}_{6} \mathrm{H}_{5} \mathrm{~N}_{3}$ : C, 60.50; H, 4.23; N, 35.27. Found: C 60.37; H 4.28; N 35.31.

\footnotetext{
${ }^{17}$ Sakamoto, T.; Ohsawa, K. J. Chem. Soc., Perkin Trans. 1, 1999, 2323

${ }^{18}$ Caldwell, W. T.; Tyson, F., T.; Lauer, L. J. Am. Chem. Soc. 1944, 66, 1479.

${ }^{19}$ Sundberg, R. J.; Biswas, S.; Murthi, K. K.; Rowe, D. J. Med. Chem. 1998, 41, 4317.
} 


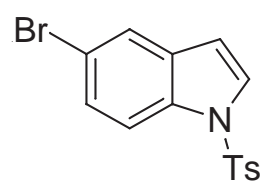

\section{5-Bromo-1-(p-toluenesulfonyl)- $1 H$-indole}

A $100 \mathrm{~mL}$ round bottom flask was sequentially charged with 5-bromoindole (1.96 g, $10 \mathrm{mmol}), p$ toluenesulfonyl chloride ( $2.30 \mathrm{~g}, 12 \mathrm{mmol})$, tetrabutylammonium hydrogen sulfate $(240 \mathrm{mg}, 0.70$ $\mathrm{mmol})$ and toluene $(40 \mathrm{~mL})$. An aqueous solution of potassium hydroxide $(13 \mathrm{~mL}, 50 \%)$ was added dropwise and the mixture was stirred at room temperature overnight. At this point the organic layer was separated, diluted with ethyl ether $(40 \mathrm{~mL})$, washed with two portions of dilute potassium hydroxide solution $(2 \times 20 \mathrm{~mL}, 2 \mathrm{M})$ and dried over $\mathrm{MgSO}_{4}$. The solvent was removed at reduced pressure and the product was purified by flash chromatography on silica gel (hexane/ethyl acetate 1:1) to provide the desired product as a light yellow solid (3.50 g, 99\% yield). Mp $135{ }^{\circ} \mathrm{C}$ (lit., ${ }^{20} 139-140{ }^{\circ} \mathrm{C}$ ). ${ }^{1} \mathrm{H}$ NMR (lit.; ${ }^{20} 400 \mathrm{MHz}, \mathrm{CDCl}_{3}, J$ values are reported in $\mathrm{Hz}): \delta 7.88(\mathrm{~d}, J=8.8,1 \mathrm{H}), 7.76(\mathrm{~d}, J=8.6,2 \mathrm{H}), 7.68(\mathrm{~d}, J=1.8,1 \mathrm{H}), 7.59(\mathrm{~d}, J=3.5,1 \mathrm{H})$, $7.42(\mathrm{dd}, J=8.8, J=2.0,1 \mathrm{H}), 7.25(\mathrm{~d}, J=8.6,2 \mathrm{H}), 2.37$ (s, 3H); ${ }^{13} \mathrm{C} \mathrm{NMR}\left(100 \mathrm{MHz}, \mathrm{CDCl}_{3}\right): \delta$ 145.7, 135.3, 133.9, 132.9, 130.4, 128.0, 127.9, 127.2, 124.5, 117.2, 115.4, 108.7, 22.0.

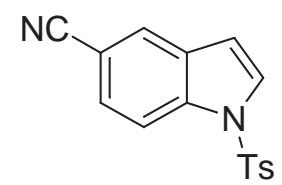

\section{1-(p-Toluenesulfonyl)-1H-indole-5-carbonitrile (Table 2, Entry 5)}

Following the general procedure, 5-bromo-1-( $p$-toluenesulfonyl)- $1 H$-indole $(607 \mathrm{mg}, 1.73 \mathrm{mmol})$ was converted into 1 -( $p$-toluenesulfonyl)-1H-indole-5-carbonitrile in $24 \mathrm{~h}$ at $110^{\circ} \mathrm{C}$. Purification of the crude product by column chromatography on silica gel (hexane/ethyl acetate 6:1) provided the desired product as a white crystalline powder (475 mg, 93\% yield). Mp 130-131 ${ }^{\circ} \mathrm{C} .{ }^{1} \mathrm{H}$ NMR (400 MHz, $\mathrm{CDCl}_{3}, J$ values are reported in $\mathrm{Hz}$ ): $\delta 8.09$ (dt, $\left.J=8.7, J=0.8,1 \mathrm{H}\right), 7.90$ (d, $J=$ $1.6,1 \mathrm{H}), 7.80(\mathrm{dt}, J=8.3, J=1.8,2 \mathrm{H}), 7.72(\mathrm{~d}, J=3.7,1 \mathrm{H}), 7.58(\mathrm{dd}, J=8.7, J=1.7,1 \mathrm{H}), 7.29(\mathrm{~d}$, $J=8.3,2 \mathrm{H}), 6.73(\mathrm{dd}, J=3.7, J=0.8,1 \mathrm{H}), 2.39(\mathrm{~s}, 3 \mathrm{H}) ;{ }^{13} \mathrm{C} \mathrm{NMR}\left(100 \mathrm{MHz}, \mathrm{CDCl}_{3}\right): \delta 146.2$, 136.8, 135.1, 131.1, 130.6, 128.8, 128.0, 127.3, 126.8, 119.8, 114.7, 108.9, 107.3, 22.1; IR (neat, $\mathrm{cm}^{-1}$ ): 2226, 1597, 1456, 1373, 1269, 1174, 1138, 672, 593, 540. Anal. Calcd. for $\mathrm{C}_{16} \mathrm{H}_{12} \mathrm{~N}_{2} \mathrm{O}_{2} \mathrm{~S}$ : C, 64.85; H, 4.08; N, 9.45. Found: C, 65.04; H, 4.11; N, 9.47.

\footnotetext{
${ }^{20}$ Fresneda, M. P.; Molina, P.; Bleda, A. J. Tetrahedron 2001, 57, 2355.
} 


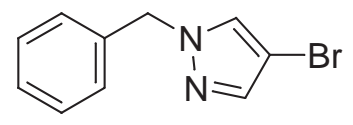

\section{1-Benzyl-4-bromo-1H-pyrazole ${ }^{21}$}

A $100 \mathrm{~mL}$ round bottom flask was charged with 4-bromopyrazole (4.41 g, $30 \mathrm{mmol}$ ), tetrabutylammonium bromide $(484 \mathrm{mg}, 1.5 \mathrm{mmol})$ and potassium hydroxide pellets $(3.37 \mathrm{~g}, 60$ mmol). After the mixture was sonicated for $15 \mathrm{~min}$, benzyl chloride $(5.2 \mathrm{~mL}, 45 \mathrm{mmol})$ was added dropwise and the resulting mixture was stirred overnight. Ethyl ether $(20 \mathrm{~mL})$, water $(20$ $\mathrm{mL})$, and diluted hydrochloric acid $(1 \mathrm{~mL}, 10 \%)$ were added under stirring. The organic layer was washed with water $(2 \times 20 \mathrm{~mL})$ and dried over $\mathrm{MgSO}_{4}$. The solvent was removed under reduced pressure and the product was purified by flash chromatography on silica gel (hexane/ethyl acetate 10:1) to provide the desired product as a white solid ( $6.74 \mathrm{~g}, 95 \%$ yield). Mp $51-52{ }^{\circ} \mathrm{C}$ (lit., ${ }^{22}$ 44-45 $\left.{ }^{\circ} \mathrm{C}\right) . \quad{ }^{1} \mathrm{H}$ NMR $\left(400 \mathrm{MHz}, \mathrm{CDCl}_{3}\right): \delta 7.53(\mathrm{~s}, 1 \mathrm{H}), 7.42-7.33(\mathrm{~m}, 4 \mathrm{H}), 7.28-7.22(\mathrm{~m}, 2 \mathrm{H})$, 5.29 (s, $2 \mathrm{H}) ;{ }^{13} \mathrm{C}$ NMR $\left(100 \mathrm{MHz}, \mathrm{CDCl}_{3}\right): \delta 140.4,136.2,129.8,129.4,128.8,128.3,93.9,57.1$.<smiles>N#Cc1cnn(Cc2ccccc2)c1</smiles>

\section{1-Benzyl-1H-pyrazole-4-carbonitrile (Table 2, Entry 6)}

Following the general procedure, 1-benzyl-4-bromo-1H-pyrazole (308 $\mathrm{mg}, 1.74 \mathrm{mmol}$ ) was converted into 1-benzyl- $1 \mathrm{H}$-pyrazole-4-carbonitrile in $24 \mathrm{~h}$ at $110^{\circ} \mathrm{C}$. Purification of the crude product by column chromatography on silica gel (hexane/ethyl acetate 5:1) provided the desired product as a light yellow solid (252 mg, 80\% yield). Mp 61-63 ${ }^{\circ} \mathrm{C} .{ }^{1} \mathrm{H}$ NMR (400 MHz, $\left.\mathrm{CDCl}_{3}\right): \delta 7.85(\mathrm{~s}, 1 \mathrm{H}), 7.78(\mathrm{~s}, 1 \mathrm{H}), 7.45-7.36(\mathrm{~m}, 2 \mathrm{H}), 7.31-7.24(\mathrm{~m}, 2 \mathrm{H}), 5.35(\mathrm{~s}, 2 \mathrm{H}) ;{ }^{13} \mathrm{C} \mathrm{NMR}$ (100 MHz, $\mathrm{CDCl}_{3}$ ): 143.1, 134.8, 134.6, 129.6, 129.3, 128.5, 113.8, 93.1, 51.2; IR (neat, $\mathrm{cm}^{-1}$ ): $3109,2231,1543,1455,1440,1383,1354,1152,1004,991,718,693$. Anal. Calcd. for $\mathrm{C}_{11} \mathrm{H}_{9} \mathrm{~N}_{3}$ : C, 72.11; H, 4.95; N, 22.94. Found: C 72.00; H 4.92, N 23.01.

\footnotetext{
${ }^{21}$ We are grateful to Michele C. Harris for performing this reaction.

${ }^{22}$ Jones, R. G. J. Am. Chem. Soc. 1949, 71, 3994.
} 


\section{Conversion of 5-Iodo-m-xylene into 3,5-Dimethylbenzonitrile Using Different Copper Precatalysts}

Three Schlenk tubes were charged with sodium cyanide $(102 \mathrm{mg}, 2.08 \mathrm{mmol})$ and either CuI (33 $\mathrm{mg}, 0.17 \mathrm{mmol}, 10 \mathrm{~mol} \%), \mathrm{CuBr}$ (25 mg, $0.17 \mathrm{mmol}, 10 \mathrm{~mol} \%$ ), or CuCN (15.5 mg, $0.173 \mathrm{mmol}$, $10 \mathrm{~mol} \%)$. The Schlenk tubes were evacuated and backfilled with argon. Toluene (1.2 mL), N,N'dimethylethylenediamine (185 $\mu \mathrm{L}, 1.74 \mathrm{mmol})$, and 5-iodo- $m$-xylene $(250 \mu \mathrm{L}, 1.73 \mathrm{mmol})$ were added to each Schlenk tube. The Schlenk tubes were sealed with Teflon valves, and the reaction mixtures were stirred at $90^{\circ} \mathrm{C}$ in an oil bath for $24 \mathrm{~h}$. The resulting suspensions were allowed to reach room temperature. Ethyl acetate $(2 \mathrm{~mL})$ and dodecane (internal $\mathrm{GC}$ standard, $200 \mu \mathrm{L}$ ) were added to the reaction mixtures. A $50 \mu \mathrm{L}$ sample of the supernatant solution was diluted with ethyl acetate $(1 \mathrm{~mL})$ and analyzed by $\mathrm{GC}$ to provide the following results:

CuI, 99.9\% conversion of 5-iodo- $m$-xylene and $96 \%$ yield of 3,5-dimethylbenzonitrile; $\mathrm{CuBr}, 99.7 \%$ conversion of 5-iodo- $m$-xylene and $97 \%$ yield of 3,5-dimethylbenzonitrile; $\mathrm{CuCN},>99.9 \%$ conversion of 5-iodo- $m$-xylene and $98 \%$ yield of 3,5-dimethylbenzonitrile.

\section{Conversion of 5-Bromo-m-xylene into 3,5-Dimethylbenzonitrile Using Different Copper Precatalysts}

Three Schlenk tubes were charged with sodium cyanide $(102 \mathrm{mg}, 2.08 \mathrm{mmol})$ and either $\mathrm{CuI}(33$ $\mathrm{mg}, 0.17 \mathrm{mmol}, 10 \mathrm{~mol} \%$ ), $\mathrm{CuBr}$ (25 mg, $0.17 \mathrm{mmol}, 10 \mathrm{~mol} \%$ ), or $\mathrm{CuCN}$ (15.5 mg, $0.173 \mathrm{mmol}$, $10 \mathrm{~mol} \%)$. The Schlenk tubes were evacuated and backfilled with argon. Toluene $(1.2 \mathrm{~mL}), N, N^{\prime}-$

dimethylethylenediamine (185 $\mu \mathrm{L}, 1.74 \mathrm{mmol})$, and 5-bromo- $m$-xylene $(235 \mu \mathrm{L}, 1.73 \mathrm{mmol})$ were added to each Schlenk tube. The Schlenk tubes were sealed with Teflon valves, and the reaction mixtures were stirred at $110^{\circ} \mathrm{C}$ in an oil bath for $24 \mathrm{~h}$. The resulting suspensions were allowed to reach room temperature. Ethyl acetate $(2 \mathrm{~mL})$ and dodecane (internal GC standard, $200 \mu \mathrm{L}$ ) were added to the reaction mixtures. A $50 \mu \mathrm{L}$ sample of the supernatant solution was diluted with ethyl acetate $(1 \mathrm{~mL})$ and analyzed by $\mathrm{GC}$ to provide the following results:

CuI, 91\% conversion of 5-bromo- $m$-xylene, $82 \%$ yield of 3,5-dimethylbenzonitrile, and $3 \%$ yield of 5-iodo- $m$-xylene; 
$\mathrm{CuBr}, 10 \%$ conversion of 5-bromo- $m$-xylene and $2 \%$ yield of 3,5-dimethylbenzonitrile; $\mathrm{CuCN}, 5 \%$ conversion of 5-bromo-m-xylene and $1 \%$ yield of 3,5-dimethylbenzonitrile. 